TRANSACTIONS OF THE

AMERICAN MATHEMATICAL SOCIETY

Volume 214, 1975

\title{
EQUISINGULAR DEFORMATIONS OF PUISEUX EXPANSIONS
}

\author{
BY
}

\begin{abstract}
AUGUSTO NOBILE
ABSTRACT. Parametrizations of a deformation (over a complete local ring) of an irreducible algebroid curve are studied. With these parametrizations another definition of equisingularity (equivalent to the known ones) is given, by using their characteristic numbers. These methods are also used in the complex analytic case. The following applications of these techniques are given: a proof of the existence of a versal equisingular deformation in the complex analytic case; a proof that an equisingular formal family of branches is determined by its $\nu$-truncation ( $\nu$ large enough, depending only on the characteristic of the special fiber).
\end{abstract}

Introduction. We study certain questions of the theory of equisingular deformations of an irreducible plane curve. The theory of equisingularity (or equivalence) for plane curves was initiated (at least in modern language) by Zariski [15, I and II]. In the irreducible case, a possible approach to this theory is the use of parametrizations (or Puiseux expansions) of a given branch and their characteristic pairs (or numbers); see [15, III, §3], [16, §4]. Thus, a natural approach to studying equisingular deformations of a branch is by using parametrizations, but defined over rings rather than a field. Probably several people considered this approach; for instance in a Harvard University seminar in the spring of 1972, Mumford presented some basic definitions of an equisingular deformation theory based on Puiseux expansions and proved that the corresponding functor is isomorphic to Wahl's functor (cf. [13]; here we present a different proof). However, as far as we know, no systematic study of parametrizations over complete local rings has been presented, and that is what we do in $\S \S 1$ and 2. The theory has some nice features; for instance it allows us to make very explicit computations (hopefully this could help to better understand certain problems, like questions of prorepresentability, automorphisms of deformations, dimensions, etc.), and the convergent case can be treated with similar methods. In $\S 4$ we discuss the complex analytic case. As an application of these methods, we give, in $\S 3$, an elementary proof that a formal equisingular family of branches (cf. Definition 3.1) is determined by its $\nu$-trunca-

Received by the editors June 7, 1973.

AMS (MOS) subject classifications (1970). Primary 14H20, 32B99; Secondary 14H99, 32G05.

Key words and phrases. Branch, Puiseux expansion, deformation parametrization, characteristic of a parametrization, equisingular deformation, versal deformation, formal family of branches, $\nu$-truncation of a family. 
tion for $\nu$ large enough and depending only on the characteristic of the special curve. In $\$ 4$ there is another application: we give a simple proof of the existence of a versal complex analytic equisingular deformation of a branch which does not use the convergence arguments that are common in theorems of this type.

The influence on this paper of Zariski's work on equisingularity is obvious. Aside from his published articles, we found extremely helpful some of his oral expositions on the subject.

Finally, we want to thank the referee for a large number of suggestions and corrections on a previous version of this work. (There are too many to enumerate.)

0 . Notations and terminology. Throughout this work, $k$ will be an algebraically closed field of characteristic zero. The category of complete local $k$-algebras, with residue field $k$, with the usual $k$-algebra homomorphisms (which are necessarily local) as morphisms, will be donoted by $A_{c}$; its full subcategory of finite dimensional algebras (as $k$-vector spaces) by $\mathrm{A}$. Given a category $\mathrm{C}$, the class of objects of $C$ will also be denoted by $C$. Given a local ring $A, r(A)$ denotes the maximal ideal of $A$. The ring of formal power series in the variables $x_{1}, \ldots, x_{n}$ with coefficients in the ring $A$ is denoted by $A\left[\left[x_{1}, \ldots, x_{n}\right]\right]$.

Given a power series $\varphi$, its order is denoted by $o(\varphi)$. If $\varphi=\Sigma_{i=m}^{\infty} b_{i} t^{i}$, where $b_{m} \neq 0$, then we say that $b_{m}$ is the initial coefficient of $\varphi$. If $A \in A_{c}, f \in$ $A\left[\left[x_{1}, \ldots, x_{n}\right]\right]$, then $\operatorname{res}(f)$ denotes the power series in $k\left[\left[x_{1}, \ldots, x_{n}\right]\right]$ obtained from $f$ by replacing each coefficient by its image (under the canonical homomorphism) in $k$. Given $\varphi \in A[[t]]$, its reduced order, denoted by $\bar{o}(\varphi)$, is the order of res $(\varphi)$. Thus, $o(\varphi)=\bar{o}(\varphi)$ means that the initial coefficient of $\varphi$ is a unit.

Given an irreducible power series $f_{0} \in k[[x, y]]$, the scheme $X_{0} \doteq$ $\operatorname{Spec}\left(k[[x, y]] /\left(f_{0}\right)\right)$ is called an algebroid branch, or simply a branch. Sometimes, in an abuse of language, we shall call the ring $k[[x, y]] /\left(f_{0}\right)$, itself, a branch, or we may even talk about "the branch $f_{0}$ ".

1. Deformations and parametrizations. Throughout $\S \S 1$ and $2, f_{0}$ will denote an irreducible power series in $k[[x, y]]$.

Definition 1.1 (a). Let $A \in A_{c}$; a deformation of $f_{0}$ over $A$ is a series $f \in$ $A[[x, y]]$ such that $f(0,0)=0$ and $f_{0}=\operatorname{res}(f)$. (b) Two deformations $f, f^{\prime}$ of $f_{0}$ over $A$ are said to be similar if there are series $u, \alpha, \beta$ in $A[[x, y]]$, where $\alpha-x$, $\beta-y \in M[[x, y]](M=r(A))$ and $u$ is a unit such that

$$
u(x, y) f^{\prime}(\alpha(x, y), \beta(x, y))=f(x, y) \text {. }
$$

REMARK 1.2. The usual word in this kind of situation is "equivalent" rather than "similar". Since we shall talk later "equivalent curves" in the sense of Zariski (see $[15, \mathrm{I}]$ ) we deviate from the usual terminology to avoid confusion. 
Definition 1.3. (i) Let $f(x, y) \in A[[x, y]]$, where $A \in \mathrm{A}_{c}$. A parametrization of $f$ is a pair $(\varphi, \psi)$ of power series in $A[[t]]$ such that:

(a) $f(\varphi(t), \psi(t))=0, \varphi(0)=\psi(0)=0$.

(b) The greatest common divisor of the exponents of $t$ in $\operatorname{res}(\varphi)$ and $\operatorname{res}(\psi)$, with nonzero coefficient, is 1 ; i.e., $k[[\operatorname{res}(\varphi)$, $\operatorname{res}(\psi)]]$ is not contained in $k\left[\left[t^{s}\right]\right]$ for any $s>1$.

(ii) A parametrization $(\varphi, \psi)$ is called strict if the initial coefficients of $\varphi$ and $\psi$ are units.

Definition 1.4. Two parametrizations $(\varphi, \psi),\left(\varphi^{\prime}, \psi^{\prime}\right)$ of $f$ are called associated if $\left(\varphi^{\prime}, \psi^{\prime}\right)$ is obtained from $(\varphi, \psi)$ by a substitution $t \rightarrow \alpha(t)=d_{1} t+$ $d_{2} t^{2}+\ldots$, where $d_{1}$ is a unit.

Recall that if $A \in \mathrm{A}_{c}$ and $u$ is a unit of $A$, then, for any natural number $n$, there are $n$th roots of $u$ in $A$.

Proposition 1.5. Let $f \in A[[x, y]]$ and $(\varphi, \psi)$ a parametrization of $f$, where $o(\varphi)=\bar{o}(\varphi)=n$. Then there is an associated parametrization of the form $\left(t^{n}, \psi(t)\right)$. If $\left(t^{n}, \psi^{\prime}(t)\right)$ is another parametrization associated to $(\varphi, \psi)$, then $\psi^{\prime}(t)=\psi(w t)$, where $w$ is an $n$th root of 1 .

Proof. The proof for the case $A=k$ given in [14, p. 95, Theorem 2.2], applies without changes (all we need is the existence of $n$th roots of units of $A$ ).

Proposition 1.6. Let $A \in \mathrm{A}, f \in A[[x, y]]$ be a deformation of $f_{0},(\psi, \varphi)$ a parametrization of $f, p: A[[x, y]] \rightarrow A[[t]]$ the homomorphism satisfying $p(x)=\psi, p(y)=\varphi, P=\operatorname{Ker}(p), A[[\psi, \varphi]]=\operatorname{Im}(p)$. Then:

(a) $P=(f)$.

(b) For any ideal I of $A$, the canonical map $\alpha(I): A[[\psi, \varphi]] \otimes_{A} A / I \rightarrow$ $A / I\left[\left[\psi^{\prime}, \varphi^{\prime}\right]\right]$ (where $\psi^{\prime}\left(\right.$ resp. $\left.\varphi^{\prime}\right)$ is obtained from $\psi$ (resp. $\varphi$ ) by reducing the coefficients $\bmod I)$ is an isomorphism.

Proof. We shall prove (a) and (b) simultaneously by induction on $l=$ $\operatorname{dim}_{k} M$, where $M=r(A)$.

In the case $l=0$, i.e., $A=k$, (b) is trivial and (a) is well known, since $P$ must be a prime ideal of height one in the unique factorization domain $k[[x, y]]$. Now we need

LEMMA 1.7. The following statements are equivalent:

(a) of Proposition 1.6;

(c) $A[[x, y]] / P$ is $A$-flat.

Proof of Lemma 1.7. The implication (a) $\Rightarrow$ (c) is well known since $A[[x, y]] /(f)$ is $A$-flat $[8,20 . F]$. To show that (c) $\Rightarrow($ a), note that clearly $f \in P$, hence we have a commutative diagram: 


$$
\begin{aligned}
& 0 \rightarrow(f) \rightarrow A[[x, y]] \rightarrow A[[x, y]] /(f) \rightarrow 0
\end{aligned}
$$

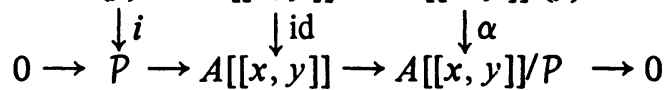

(where $i$ is an inclusion). Tensoring with $k$ (over $A$ ) we get, using the fact that $A[[x, y]] / P \approx A[[\psi, \varphi]]$ and (c), a commutative diagram with exact rows:

$$
\begin{aligned}
& 0 \rightarrow\left(f_{0}\right) \rightarrow k[[x, y]] \rightarrow(A[[x, y]] /(f)) \otimes_{A} k \rightarrow 0 \\
& \downarrow i_{0} \quad \downarrow \text { id } \quad \downarrow \alpha \otimes 1 \\
& 0 \rightarrow P \otimes k \rightarrow j k[[x, y]] \rightarrow(A[[x, y]] / P) \otimes_{A} k \rightarrow 0
\end{aligned}
$$

We claim that $i_{0}$ is an isomorphism. It is clearly injective. To see that it is onto, let $\varphi_{0}=\operatorname{res}(\varphi), \psi_{0}=\operatorname{res}(\psi)$ (note that $\left(\psi_{0}, \varphi_{0}\right)$ is a parametrization of $\left.f_{0}\right)$ and $p_{0}: k[[x, y]] \rightarrow k[[t]]$ be given by $p_{0}(x)=\psi_{0}, p_{0}(y)=\varphi_{0}$. Then we see that $\operatorname{Ker}\left(p_{0}\right)=\left(f_{0}\right)$. Obviously, $\operatorname{Pk}[[x, y]] \subseteq \operatorname{Ker}\left(p_{0}\right)=\left(f_{0}\right)$. We have

$$
\left(f_{0}\right)=j i_{0}\left(\left(f_{0}\right)\right) \subseteq j(P \otimes k)=P k[[x, y]] \subseteq\left(f_{0}\right) .
$$

Since $j$ is injective, $i_{0}$ is onto.

Then $\alpha \otimes 1$ is an isomorphism, i.e., $A[[x, y]] /(f)$ and $A[[x, y]] / P$ are isomorphic $\bmod M$. Since $M$ is a nilpotent ideal and $A[[x, y]] / P$ is $A$-flat, it follows that $\alpha$ is an isomorphism [10, Lemma 3.3]. Hence, $\left(f_{0}\right)=P$. This proves the lemma.

CONTINUATION OF THE PROOF OF 1.6. Now we prove the inductive step. Assume the assertions (a), (b) are true for any $A^{\prime}$ such that $\operatorname{dim}_{k} r\left(A^{\prime}\right)<l$. Let $A \in \mathrm{A}$, where $\operatorname{dim} r(A)=l$. First, we prove (b). Let $I$ be any ideal of $A$, set $A^{\prime}=A / I, \alpha=\alpha(I)$. It is immediate that $\alpha$ is surjective. To see that $\alpha$ is also injective, it suffices to show: given $g \in A[[x, y]]$, if its image $g^{\prime}$ in $A^{\prime}[[x, y]]$ satisfies $g^{\prime}\left(\psi^{\prime}, \varphi^{\prime}\right)=0$, then $g(\psi, \varphi)=\Sigma m_{i j} \psi^{i} \varphi^{j}$, with $m_{i j} \in I, \forall i, j$. (This follows from the fact that $A^{\prime}$ is a finite $A$-algebra. Hence $A[[\psi, \varphi]] \otimes_{A} A^{\prime}=$ $A[[\psi, \varphi]] \hat{\otimes}_{A} A^{\prime}$ and $\left(\sum_{i=0}^{\infty} g_{i}\right) \otimes 1=\Sigma_{i=0}^{\infty}\left(g_{i} \otimes 1\right)$.) Consider such a $g$. By the induction hypothesis (a), the relation $g^{\prime}\left(\psi^{\prime}, \varphi^{\prime}\right)$ implies the existence of an $h^{\prime} \in$ $A^{\prime}[[x, y]]$ such that $g^{\prime}=h^{\prime} f^{\prime}$, where $f^{\prime}$ is the image of $f$ in $A^{\prime}[[x, y]]$. Lifting to $A[[x, y]]$, we get a relation $g-h f=\Sigma m_{i j} x^{i} y^{j}, m_{i j} \in I$. By making $x=\psi, y=$ $\varphi$, we get $g(\psi, \varphi)=\Sigma m_{i j} \psi^{i} \varphi^{j}$, and (b) is true. Now we show (c) of Lemma 1.7, which is equivalent to (a). Write $B=A[[\psi, \varphi]]$; we shall see that $B$ is $A$-flat. By using the infinitesimal criterion (see $[8,20 . C]$ ), it is enough to check the following: let $\epsilon \in A, \epsilon \neq 0$ such that $\epsilon M=0, J=(\epsilon), A_{1}=A / J$; then (i) $B \otimes_{A} A_{1}$ is $A_{1}{ }^{-}$ flat, (ii) $j: J \otimes_{A} B \rightarrow J B$ is injective. To see (i), note that by (b) (which was already proved), $B \otimes_{A} A_{1}=A_{1}\left[\left[\psi_{1}, \varphi_{1}\right]\right]$ (where $\psi_{1}, \varphi_{1}$ are $\psi, \varphi$, respectively, $\bmod J$ ). But $\operatorname{dim}_{k} r\left(A_{1}\right)<l$; hence, by induction hypothesis (a) (or (c)), $B \otimes_{A}$ $A_{1}$ is $A_{1}$-flat. To check (ii), consider an element of $\operatorname{Ker}(j)$. It can be written as $\epsilon \otimes g(\psi, \varphi), g \in A[[x, y]]$, where $\epsilon g(\psi, \varphi)=0$. Write $g=\Sigma\left(c_{i j}+m_{i j}\right) x^{i} y^{j}$, 
where $c_{i j} \in k, m_{i j} \in M$. Let $q(t)=\Sigma c_{i j} \psi^{i} \varphi^{j} \in A[[t]]$. The condition $\in M=0$ implies $0=\epsilon g(\psi(t), \varphi(t))=q(t)$; hence $\operatorname{res}(q)=0$, i.e., all the coefficients of $q$ are in $M$. Then, writing $\varphi_{0}=\operatorname{res}(\varphi), \psi_{0}=\operatorname{res}(\psi), \Sigma c_{i j} \psi_{0}^{i} \varphi_{0}^{j}=\operatorname{res}(q)=0$. By induction (actually by (a) in the case $A=k$ ), we have $0=\Sigma c_{i j} x^{i} y^{j}-$ $f_{0}(x, y) h_{0}(x, y)$; lifting this to $A[[x, y]]$ we get $\Sigma c_{i j} x^{i} y^{j}-f(x, y) h(x, y)=$ $\Sigma r_{i j} x^{i} y^{j}$, where $r_{i j} \in M, \forall i j$. By making $x=\psi, y=\varphi$, we get $\Sigma c_{i j} \psi^{i} \varphi^{j}=$ $\Sigma r_{i j} \psi^{i} \varphi^{j}$. Hence, $g(\psi, \varphi)=\Sigma s_{i j} \psi^{i} \varphi^{j}$, where $s_{i j}=r_{i j}+m_{i j} \in M, \forall i j$. But $(\epsilon) \otimes_{A}$ $B$ is a finite $B$-module. As $B$ is complete, $(\epsilon) \otimes_{A} B$ is separated in the $N$-adic topology $(N=r(A))$. Hence $\epsilon \otimes g(\psi, \varphi)=\Sigma_{i, j}\left(s_{i j} \epsilon \otimes, \psi^{i} \varphi^{j}\right)=0$ (because $\epsilon M=0$ ).

Thus, both (a) and (b) hold for $A$ and the propostition is proved.

Proposition 1.8. Let $A \in A_{c}$, I an ideal of $A$ such that $A^{\prime}=A / I \in A, f a$ deformation of $f_{0}$ over $A,(\psi, \varphi)$ a parametrization of $f$, and $\psi^{\prime}$ (resp. $\left.\varphi^{\prime}\right) \psi$ (resp. $\varphi) \bmod I$. Then there is a canonical isomorphism $A[[\psi, \varphi]] \otimes_{A} A^{\prime} \stackrel{\sim}{\longrightarrow}$ $A^{\prime}\left[\left[\psi^{\prime}, \varphi^{\prime}\right]\right]$.

Proof. Apply the argument of the proof of (b) in the inductive step of 1.6, taking into account that $1.6\left(\right.$ a) holds for $A^{\prime}\left[\left[\psi^{\prime}, \varphi^{\prime}\right]\right]$, because $A^{\prime} \in A$.

THEOREM 1.9. We keep the notations and assumptions of Proposition 1.5, except that now $A$ is assumed to be in $A_{c}$. Then the following (equivalent) statements hold:

(a) $A[[x, y]] / P$ is $A$-flat.

(b) $P=(f)=$ ideal generated by $f$.

Proof. First, we see the equivalence of (a) and (b). The implication (b) $\Rightarrow$ (a) is well known (see Lemma 1.7). To see that (a) implies (b) we consider the exact sequence of $A$-modules, $0 \rightarrow K \rightarrow B /(f) \rightarrow B / P \rightarrow 0$, where $B=$ $A[[x, y]]$. After tensoring with $A / M^{i}$, where $M=r(A)$ and $i$ any natural number, the sequence remains exact (since $B / P$ is $A$-flat). But, by 1.7 and 1.8 , the new sequence is isomorphic to

$$
0 \rightarrow K / M^{i} K \rightarrow A / M^{i}\left[\left[\psi_{i}, \varphi_{i}\right]\right] \stackrel{\sim}{\longrightarrow} A / M^{i}\left[\left[\psi_{i}, \varphi_{i}\right]\right] \rightarrow 0
$$

(where $\psi_{i}, \varphi_{i}$ are the canonical images of $\psi, \varphi$ respectively). Hence $K / M^{i} K=0$. It follows that $K^{*}=0$, where $K^{*}$ is the completion of $K$ with respect to $\left\{M^{i} K\right\}$, $i=1,2, \ldots$. But if $N=r(B)$, then $\bigcap_{i=1}^{\infty} N^{i} K=(0)$ (since $B$ is complete, hence a Zariski ring). Hence $K$ is separated in the $M$-topology, and $K \subset K^{*}=(0) \mathrm{im}$ plies $K=0$, i.e., $P=(f)$.

To show (a), by the infinitesimal criterion it suffices to check that the $A / M^{i}$. modules $B \otimes_{A} A / M^{i}$ are flat for all $i \geqslant 1$. But (calling $A_{i}=A / M^{i+1}$ ) we have, by 1.8 , isomorphisms $B \otimes A_{i} \cong A_{i}\left[\left[\psi_{i}, \varphi_{i}\right]\right]$. Since $A_{i} \in A$ for all $i \geqslant 0$, we are done by Proposition 1.6(a).

REMARK 1.10. Let $A \in \mathrm{A}_{c}, \varphi(t)$ be a power series in $A[[t]]$ without constant 
term, $n$ a positive integer. Consider $\Pi_{i=1}^{n}\left(y-\varphi\left(\epsilon^{i} t\right)\right)$, where $\epsilon$ is a primitive $n$th root of 1 . Then clearly this series is of the form $h\left(t^{n}, y\right) \in A\left[\left[t^{n}, y\right]\right]$. Let $x=$ $t^{n}$. The series $h(x, y)$ is called the series associated to $\left(t^{n}, \varphi(t)\right)$. If $\left(t^{n}, \varphi\right)$ satisfies Definition $1.2(\mathrm{~b})$, then clearly $\left(t^{n}, \varphi\right)$ is a parametrization of $h$. Note also that $h(x, y)=y^{n}+B_{1}(x) y^{n-1}+\ldots+B_{n}(x), B_{i}(x) \in A[[x]], B_{i}(0)=0, i=$ $1, \ldots, n$. Moreover, if $\left(t^{n}, \varphi\right)$ is a parametrization of $f$, where $f$ is a deformation of $f_{0}$, then, by Theorem 1.9 , there is a unit $u \in A[[x, y]]$ satisfying $u f=h$.

From this and the Weierstrass preparation theorem, it follows that $h$ is the unique Weierstrass pseudopolynomial associated to $f$. In particular, if $\left(t^{m}, \varphi^{\prime}\right)$ is another parametrization of $f$, then $n=m=$ degree of the pseudopolynomial associated to $f$.

In the following proposition, we are indebted to the referee for a simplification of its proof.

Proposition 1.11. Let $A \in A_{c}, f \in A[[x, y]]$ be a deformation of $f_{0}$, $(\Gamma, \varphi)$ a parametrization $f$, where $o(\Gamma)=\bar{o}(\Gamma)$. Then any other parametrization $(N, \psi)$ of $f$, where $o(N)=\bar{o}(N)$, is associated to $(\Gamma, \varphi)$.

Proof. By Proposition 1.5 and Remark 1.10 we may assume $\Gamma=N=t^{n}$. Note that 1.11 is an easy consequence of 1.10 if $A$ is an integral domain; in fact, if $w$ is a primitive $n$th root of 1 , then $0=f\left(t^{n}, \psi(t)\right)=\Pi_{i=1}^{n}\left(\psi(t)-\varphi\left(w^{i} t\right)\right)$ in the domain $A[[t]]$, hence $\psi(t)=\varphi\left(w^{i} t\right)$, for some $i$.

For the general proof, we shall show that there is an $n$th root $w$ of 1 , such that $\varphi(w t)=\psi(t)$. Consider two cases:

Case 1. $A \in A$, i.e., $\operatorname{dim}_{k} r(A)<\infty$. We induct on $l=\operatorname{dim}_{k} r(A)$. For $l=0$ we have $A=k$, an integral domain, and we see that the assertion is true. For the inductive step, given $A \in A$, consider a small extension $p: A \rightarrow A^{\prime}$, i.e., $p$ is onto, $\operatorname{Ker}(p)=I=(\epsilon)$, where $\epsilon r(A)=0$. Let $\varphi^{\prime}\left(\right.$ resp. $\left.\psi^{\prime}\right)$ be induced by $\varphi$ (resp. $\left.\psi\right)$ in $A^{\prime}[[t]]$. By induction we have $\varphi^{\prime}(w t)=\psi^{\prime}(t)$, where $w \in k, w^{n}=1$. Let $\varphi_{1}(t)=\varphi(w t)$; we show $\varphi_{1}=\psi$. But $\varphi_{1}-\psi=\epsilon a, a \in k[[t]]$ because $\varphi_{1} \equiv$ $\psi \bmod I$, and $\epsilon M=0$. Hence, a simple computation gives

$$
0=f\left(t^{n}, \varphi_{1}\right)=f\left(t^{n}, \psi+\epsilon a\right)=\epsilon a(\partial / \partial y) f_{0}\left(t^{n}, \psi_{0}\right),
$$

where $\psi_{0}=\operatorname{res}(\psi)$. But $\partial f_{0}\left(t^{n}, \psi_{0}\right) / \partial y \neq 0$, since $\left(t^{n}, \psi_{0}\right)$ is a parametrization of the branch $f_{0}$. Hence $a=0$, i.e., $\varphi_{1}=\psi$.

Case 2. $A \in \mathrm{A}_{c}$. Let $M=r(A)$. By Case 1 , there is an $n$th root $w$ such that $\varphi(w t) \equiv \psi(t)\left(\bmod M^{m}\right), \forall m$. Hence, $\varphi(w t)=\psi(t)$ by Krull's theorem.

The following is an easy consequence of 1.11 , and we omit the proof.

Corollary 1.12. Let $A, f,(\Gamma, \varphi)$ be as in $1.11, I$ an ideal of $A, A^{\prime}=A / I$, $f^{\prime}$ the deformation over $A^{\prime}$ induced by $f$. Then any parametrization $\left(N^{\prime}, \psi^{\prime}\right)$ of $f^{\prime}$, where $o\left(N^{\prime}\right)=\bar{o}\left(N^{\prime}\right)$, can be lifted to a parametrization of $f$. 
2. Equisingular deformations. The classical definition of the characteristic of a parametrization over a field can be applied to the case of rings.

Definition 2.1 [16, p. 289]. Let $A \in A_{c}, f \in A[[x, y]],\left(t^{n}, \psi\right), \psi=$ $\Sigma a_{i} t^{i}$, be a parametrization of $f$. The characteristic of $\left(t^{n}, \psi\right)$, denoted $\operatorname{ch}\left(t^{n}, \psi\right)$, is the sequence of numbers $\left(n ; \beta_{1}, \ldots, \beta_{g}\right)$, where the $\beta_{i}$ are defined by the following conditions:

(a) $\left.\left.n\rangle\left\langle n, \beta_{1}\right\rangle\right\rangle \ldots\right\rangle\left\langle n, \beta_{1}, \ldots, \beta_{g}\right\rangle=1$ (where \langle\rangle denotes greatest common divisor).

(b) $a_{\beta_{j}} \neq 0, j=1, \ldots, g$.

(c) $\beta_{j}$ is minimum, i.e., if $\beta_{1}, \ldots, \beta_{j-1}$ have been defined, and $\left\langle n, \beta_{1}, \ldots\right.$, $\left.\beta_{j-1}\right\rangle>1$, then $\beta_{j}$ is the smallest $q$ such that $a_{q} \neq 0$ and $\left.\left\langle n, \beta_{1}, \ldots, \beta_{j-1}\right\rangle\right\rangle$ $\left\langle n, \beta_{1}, \ldots, \beta_{j-1}, q\right\rangle$.

In the classical case (when the base is a field), an important result about characteristics is the inversion formula. This result remains valid when we replace a field by $A \in A_{c}$. We have

THEOREM 2.2 (INVERSION FORMULA). Let $f \in A[[x, y]],(\varphi, \psi)$ a parametrization of $f, \varphi=a_{n} t^{n}+\ldots, \psi=b_{m} t^{m}+\ldots, a_{n}, b_{m}$ units, let $\left(t^{n}, \psi_{1}\right)$, $\left(\varphi_{1}, t^{m}\right)$ be associated to $(\varphi, \psi)$, and $\left(n ; \beta_{1}, \ldots, \beta_{g}\right),\left(m ; \beta_{1}^{\prime}, \ldots, \beta_{h}^{\prime}\right)$ be their respective characteristics. Then: (a) if $n<m<\beta_{1}$, then $h=g+1, \beta_{1}^{\prime}=n$, $\beta_{\nu+1}^{\prime}=\beta_{\nu}+n-m, \nu=1, \ldots, g$; (b) in all other cases, $g=h$ and $\beta_{\nu}^{\prime}=\beta_{\nu}+$ $n-m, \nu=1, \ldots, g$.

Proof. The proof given by Abhyankar [2] applies without changes. In fact, that proof works when we replace a field by a ring having the property that given a unit $u$ and a positive integer $p$, then $u^{1 / p}$ exists in the ring. Any $A \in A_{c}$ has this property.

For the rest of $\S 2$ we shall assume that the $y$-axis is not tangent to $X_{0}=$ Spec $k[[x, y]] /\left(f_{0}\right)$, i.e., that $o\left(f_{0}(x, y)\right)=o\left(f_{0}(0, y)\right)=n$. This is not a loss of generality since $f_{0}$ is irreducible and, hence, has only one tangent line; so by interchanging $x$ and $y$ (if necessary) we may make the above assumption.

Definition 2.3. Assume $f$ is a deformation of $f_{0}$ to $A \in A_{c}$ which admits a parametrization $(\varphi, \psi)$. We shall say that the parametrization is equisingular if:

(a) $n=o\left(f_{0}\right)=o(\varphi)=\bar{o}(\varphi) \leqslant o(\psi)$.

(b) There is a parametrization $\left(t^{n}, \psi^{\prime}\right)$ associated with $(\varphi, \psi)$ such that $\operatorname{ch}\left(t^{n}, \psi^{\prime}\right)=\operatorname{ch}\left(t^{n}, \operatorname{res}\left(\psi^{\prime}\right)\right)$

In this definition, apparently one is giving a privileged role to the variable $x$. If also $o(\psi)=\bar{o}(\psi)=n$, this is not reasonable. But one easily sees by using the inversion formula that if this is the case, then $(\varphi, \psi)$ is equisingular, in the sense of 2.3 , if and only if there is a parametrization $\left(\varphi^{\prime}, t^{n}\right)$ associated with $(\varphi, \psi)$ 
such that $\operatorname{ch}\left(\phi^{\prime}, t^{n}\right)=\operatorname{ch}\left(\operatorname{res}\left(\varphi^{\prime}\right), t^{n}\right)$.

Note that by Proposition 1.11, if a deformation admits an equisingular parametrization $\left(t^{n}, \varphi\right)$; then any other parametrization $\left(t^{n}, \varphi^{\prime}\right)$ will be equisingular of the same characteristic.

Definition 2.4. (a) A deformation $f$ of $f_{0}$ over $A \in A_{c}$ is called $P$-equisingular if it admits an equisingular parametrization.

(b) If $f$ is a $P$-equisingular deformation of $f_{0}$, the characteristic of $f$ is $\operatorname{ch}\left(t^{n}, \varphi\right)$, where $\left(t^{n}, \varphi\right)$ is any equisingular parametrization of $f$.

LEMma 2.5. Given $A^{\prime} \in \mathrm{ob}\left(\mathrm{A}_{c}\right), A=A^{\prime} / I$ (a quotient of $\left.A^{\prime}\right), f \in A[[x, y]]$ a deformation of $f_{0}$ admitting a parametrization $\left(t^{n}, \varphi\right)$, there is a deformation $f^{\prime} \in A^{\prime}[[x, y]]$ of $f_{0}$, parametrized by $\left(t^{n}, \varphi^{\prime}\right)$, such that $f^{\prime}$ and $\varphi^{\prime}$ reduce to $f$ and $\varphi \bmod I$ respectively, and $o(\varphi)=o\left(\varphi^{\prime}\right)$. If $\left(t^{n}, \varphi\right)$ is equisingular, $\left(t^{n}, \varphi^{\prime}\right)$ can be taken equisingular.

ProOF. Let $h=\Pi_{i=1}^{n}\left(y-\varphi\left(\epsilon^{i} x^{1 / n}\right)\right)(\epsilon$ a primitive $n$th root of 1$)$ be the series associated to $\left(t^{n}, \varphi\right)$. Then $u h=f$ is a unit by 1.9 and 1.10. Lift $u, \varphi=$ $\Sigma a_{i} t^{i}$ to power series $u^{\prime}, \varphi^{\prime}=\Sigma a_{i}^{\prime} t^{i}$ over $A^{\prime}$, taking care to take $a_{i}^{\prime}=0$ if $a_{i}=0$. Then $f^{\prime}=u^{\prime} h^{\prime}$ (where $h^{\prime}$ is associated with $\left(t^{n}, \varphi^{\prime}\right)$ ) and $\varphi^{\prime}$ clearly satisfy the requirements.

REMARK 2.6. Note that if $A \rightarrow A^{\prime}$ is a homomorphism in $A_{c}$ and $f \in$ $A[[x, y]]$ is a $P$-equisingular deformation of $f_{0}$ to $A$, there is a naturally induced equisingular deformation $f^{\prime}$ of $f_{0}$ over $A^{\prime}$ defined in an obvious way.

Now we proceed to compare Definition 2.4 with other definitions of equisingularity.

REMARK 2.7. (a) Zariski has defined a notion of equisingularity of deformations (which we shall call $Z$-equisingularity) valid for $f_{0}$ not necessarily irreducible, but restricted to the case when the "parameter ring" $A$ is regular. We recall the definition in the case of a branch $f_{0}$ (see [16]). Assume $f$ is a deformation of $f_{0}$ over $A, o\left(f_{0}(x, y)\right)=o\left(f_{0}(0, y)\right)$, i.e., $x$ is a transversal parameter and $A \in \mathrm{A}_{c}$ is regular. Let $X=\operatorname{Spec}(A[[x, y]] /(f)), Y=\operatorname{Spec}(A[[x]]), p: X \rightarrow Y$ the projection induced by $A[[x]] \rightarrow A[[x, y]] /(f)$. Then the following assertions are equivalent.

(i) The branch locus of $p$ equals $V(x)$, the set of zeros of the parameter $x$.

(ii) The characteristic of the branches defined by $f_{0} \in k[[x, y]]$ and $f \in$ $K[[x, y]]$ ( $K$ an algebraic closure of $A$ ) are the same. (The characteristic of a branch $g$ [with the $y$-axis not tangent to it] is $\operatorname{ch}\left(t^{n}, \varphi\right)$ for any parametrization $\left(t^{n}, \varphi\right)$ of $g$. It is a well-defined notion [16, §4]. Thus, (ii) says that the "special curve" $f_{0}$ and the "generic curve" $f$ are equivalent [as curves defined over $K$ ]. See [16] for details.) 
(b) J. Wahl [13], has introduced another notion of equisingularity along a section (valid for $f_{0}$ not necessarily irreducible, and any $A \in \mathrm{A}_{c}$ ) essentially by requiring, in a suitable sense, equimultiplicity of the original deformation of its "reduced total transform", of the reduced total transform of this, etc. Actually, we do not need the precise definition, but the following two results found in [13] (from now on $W$-equisingular will mean equisingular, in Wahl's sense, along the trivial section).

(i) Keeping the notations of (a), if $f$ is a deformation of $f_{0}$ over a regular ring $A$, then $f$ is $W$ equisingular if and only if it is $Z$ equisingular.

(ii) Given $A^{\prime}=A / I \quad\left(A^{\prime}, A\right.$ in $\left.A_{c}\right)$ and a $W$-equisingular deformation $f^{\prime}$ of $f_{0}$ over $A^{\prime}$, then $f^{\prime}$ can be lifted to a Wequisingular deformation $f$ of $f_{0}$ over $A$.

THEOREM 2.8. Let $f$ be a deformation of $f_{0}$ over $A \in A_{c}$. Then $f$ is $P$-equisingular if and only if it is W-equisingular.

Proof. Consider first the case in which $A$ is regular. Then, by 2.7(b)(i), the notions of $W$ - and $P$ equisingularity coincide. If $f$ is $P$ equisingular, then it immediately follows that the curves $f_{0}$ and $f \in K[[x, y]]$ ( $K$ an algebraic closure of $A$ ) have the same characteristic; hence, $f$ is $Z$-equisingular. Assume now $f$ is $Z$ equisingular. We may assume, after interchanging $x$ and $y$ if necessary, that $o\left(f_{0}(x, y)\right)=o\left(f_{0}(0, y)\right)=n$. By 2.7(a)(i) we conclude, using Abhyankar's lemma [1, Theorem 4], that the normalization of $A[[x]]$ in the field of fractions of $B=A[[x, y]] /(f)$ is $A[[x]][t]$, where $t^{n}=x$, and clearly contains $B$. Then we obtain a parametrization $x=t^{n}, y=\Sigma b_{i} t^{i}, b_{i} \in A$. It is elementary to see that the assumption of $Z$-equisingularity (see $2.7(\mathrm{a})(\mathrm{ii})$ ) implies that this is an equisingular parametrization, in the sense of (2.3). Hence, $f$ is $P$ equisingular.

Consider now the general case. Let $f$ be a $W$ equisingular deformation of $f_{0}$ over $A \in \mathrm{A}_{c}$. Write $A=R / I$, where $R \in \mathrm{A}_{c}$ and is regular. Lift $f$ to a $W$-equisingular deformation $g$ of $f_{0}$ over $R$. By the regular case, $g$ is $P$ equisingular. Hence its pull-back $f$ is $P$ equisingular. The other implication is similar (using Lemma 2.5 and the fact that the pull-back of a $W$-equisingular deformation is $W$-equisingular). Theorem 2.8 is proved.

By Remark 2.6, given a branch $f_{0}$ we may define a functor $P: A_{c} \rightarrow$ (Sets) by

$$
P(A)=\left\{P \text {-equisingular deformations of } f_{0}\right\} / \text { similarity. }
$$

By Theorem 2.8, together with the fact that any deformation of $f_{0}$, equisingular in Wahl's sense, is similar to one which is equisingular along the trivial section (see [13]), it follows that $P$ is isomorphic to Wahl's equisingular deformations functor. Since this has a smooth hull, it follows:

COROLlaRy 2.9. P has a smooth hull. 
REMARK 2.10. It is not difficult to give a direct proof of 2.9 , independent of [13], by using Schlessinger's criterion. See also Remark 5.8 where a versal deformation is constructed with other methods.

To finish this section, we shall extend to our situation another classical concept of the theory of branches. Let $B=k[[x, y]],\left(t^{n}, \varphi\right)$ be a parametrization of $f_{0}$ (then $\left.B /\left(f_{0}\right) \approx k\left[\left[t^{n}, \varphi\right]\right] \subset k[[t]]\right)$. The set $\{m \in Z / m=o(g), g \in$ $\left.k\left[\left[t^{n}, \varphi\right]\right]\right\}$ is a semigroup independent of the chosen parametrization of $f_{0}$; we denote it by $S(B)$. It is also known that this semigroup depends only on the characteristic of $f_{0}$; i.e., if $f_{0}$ and $f_{0}^{\prime}$ have the same characteristic, then $S\left(B / f_{0}\right)=$ $S\left(B / f_{0}^{\prime}\right)$. In particular, if $f_{0} \in k[[x, y]]$ and $k \subset K$, another algebraically closed field, then $S\left(k[[x, y]] /\left(f_{0}\right)\right)=S\left(K[[x, y]] /\left(f_{0}\right)\right)$.

Thus given a characteristic $c=\left(n ; \beta_{1}, \ldots, \beta_{g}\right)$ (i.e., a sequence of numbers such that for some branch $f_{0}$, c is the characteristic of $f_{0}$ ), we can define $S(\mathrm{c})$ in an obvious way.

Similarly, given a deformation $f$ of $f_{0}$ over $A$, with a parametrization $\left(t^{n}, \varphi\right)$, consider the semigroup $\left\{m \in Z / m=o(g)=\bar{o}(g)\right.$, some $\left.g \in A\left[\left[t^{n}, \varphi\right]\right]\right\}$. As a consequence of Proposition 1.11, this semigroup is independent of the parametrization and is denoted $S(A[[x, y]] /(f))$.

THEOREM 2.11. Let $f$ be an equisingular deformation of $f_{0}$ over $A \in \operatorname{ob}\left(A_{c}\right)$. Then $S(A[[x, y]] /(f))=S\left(k[[x, y]] /\left(f_{0}\right)\right)$.

Proof. Call $B_{0}=k[[x, y]] /\left(f_{0}\right), B=A[[x, y]] /(f)$. Let $\left(t^{n}, \varphi\right)$ be an equisingular parametrization of $f$; then $\left(t^{n}, \varphi_{0}\right)\left(\varphi_{0}=\operatorname{res}(\varphi)\right)$ is a parametrization of $f_{0}$. The inclusion $S(B) \subseteq S\left(B_{0}\right)$ is clear from the definitions.

To see that the equality holds, note that we may assume $A$ regular. In fact, assume the result true for regular rings, then write $A=R / I, R$ regular, and lift $f,\left(t^{n}, \varphi\right)$ to $g,\left(t^{n}, \varphi^{\prime}\right)$, where $g$ is an equisingular deformation of $f_{0}$ over $R$ and $\left(t^{n}, \varphi^{\prime}\right)$ is a parametrization of $g$. Then we get a diagram of subsets of $Z$,

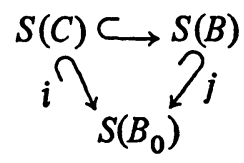

where $C=R[[x, y]] /(g)$. By our assumption, $i$ is an equality, hence, $j$ must also be an equality.

So, assume $A$ regular, of maximal ideal $M$. By contradiction suppose $S_{1}=$ $S\left(B_{0}\right) \backslash S(B) \neq \varnothing$, and let $m$ be the first element of $S_{1}$. Since $m \in S\left(B_{0}\right)$, there is $h(x, y) \in k[[x, y]]$ such that $\bar{g}=h\left(t^{n}, \varphi_{0}\right)=t^{m}+$ higher. Since $k \subset A$, we can view $h$ as an element of $A[[x, y]]$ and take

$$
g=h\left(t^{n}, \varphi\right)=a_{m_{1}} t^{m_{1}}+\text { higher, } \quad a_{m_{1}} \neq 0 .
$$


We have $m_{1} \leqslant m$ and, since $g \equiv \bar{g}(\bmod M), a_{m}$ is a unit. If $m=m_{1}$, then $m \in$ $S(B)$, a contradiction, and we are done. So assume $m_{1}<m$. Consider the generic curve of the family defined by $f($ i.e., $\operatorname{Spec}(B) \rightarrow \operatorname{Spec}(A)$ ) defined over the field $K$, an algebraic closure of $A$, by the equation $f=0$ (we follow the terminology of $[15, \mathrm{II}])$. (1) shows that $m_{1} \in S(K[[x, y]] / f)$. But by Theorem 2.8 and Remark 2.7(a), the branches $K[[x, y]] /(f)$ and $K[[x, y]] /\left(f_{0}\right)$ are equivalent. Hence, they have the same semigroup, and $m_{1} \in S\left(K[[x, y]] / f_{0}\right)=S\left(B_{0}\right)$. Since $m_{1}<m$, by minimality of $m$ we have $m_{1} \in S(B)$. Thus, we can find $g_{1} \in B$, $g_{1}=t^{m_{1}}+\ldots$ Hence $g-a_{m_{1}} g_{1}=\left(b_{m_{2}} t^{m_{2}}+\ldots\right) \in B, m_{2}<m_{1}$. Note that the coefficient of $t^{m}$ is a unit because $a_{m_{1}} \in M$; hence $m_{2} \leqslant m$. If $m_{2}=$ $m$, we have a contradiction. If $m_{2}<m$, we proceed as before to get $g_{2} \in B$, $g_{2}=t^{m_{2}}+\ldots$. Continuing in this way, after a finite number of steps we arrive at a series $t^{m}+\ldots \in B$, a contradiction.

Recall that given a subring $B$ of $k[[t]]$ (such that $k[[t]]$ is a finite $B$-module) the conductor of $B$ in $k[[t]]$ (i.e. the set of elements $b$ of $B$ such that $b k[[t]] C$ $B)$ is of the form $\left(t^{d}\right) k[[t]]$ for some integer $d \geqslant 0$. This number $d$ is called the degree of the conductor. If $B_{0}=k\left[\left[t^{n}, \varphi_{0}\right]\right]$, where $\left(t^{n}, \varphi_{0}\right)$ is a parametrization of the branch $f_{0}$, then $d$ depends only on the characteristic of the branch (see, e.g., [4, Introduction]).

Thus, we may talk about the "degree of the conductor" of a characteristic $\mathbf{c}=\left(n ; \beta_{1}, \ldots, \beta_{g}\right)$. Working over rings we have

COROLlary 2.12. Let $f$ be as in Theorem $2.11,\left(t^{n}, \varphi\right)$ a parametrization of $f, \mathrm{c}=\left(n ; \beta_{1}, \ldots, \beta_{g}\right)=\operatorname{ch}\left(t^{n}, \varphi\right), d$ the degree of the conductor of $\mathrm{c}$. If $\alpha \in$ $A[[t]]$ and $o(\alpha) \geqslant d$, then $\alpha \in A\left[\left[t^{n}, \varphi\right]\right]$.

Proof. Write $B_{0}=k\left[\left[t^{n}, \varphi_{0}\right]\right]\left(\varphi_{0}=\operatorname{res}(\varphi)\right)$ and $B=A\left[\left[t^{n}, \varphi\right]\right]$. Let $\alpha(t)=\Sigma_{i=m}^{\infty} a_{i} t^{i}, a_{m} \neq 0$. Clearly $m \in S\left(B_{0}\right)$, since $m \geqslant d$. Hence, by Theorem 2.11, $m \in S(B)$. Hence, there is $\alpha_{m}=a_{m} t^{m}+\ldots \in B$. We write $\alpha=\alpha_{m}+$ $\beta_{m+1}$; then $o\left(\beta_{m+1}\right) \geqslant m+1$. Repeating this process and using the completeness of $B$, we see that $\alpha \in B$.

COROLlary 2.13 ([3, Lemma 1]). We keep the notations of Corollary 2.12 .

(a) Then there is a unique polynomial $P \in A[t]$ of the form $P(t)=t^{\beta_{1}}+$ $\Sigma_{i \in R} b_{i} t^{i}$, where $R=\left\{i \in Z / \beta_{1}<i<d, i \notin S(\mathrm{c})\right\}$ such that $A\left[\left[t^{n}, \varphi(t)\right]\right]=$ $A\left[\left[t^{n}, P(t)\right]\right]$.

(b) There is an automorphism $\phi$ of $A[[x, y]]$ such that $\left(t^{n}, P\right)$ is a parametrization of $\phi(f)$.

Proof. Let $\varphi(t)=\Sigma a_{i} t^{i}$. Then, since $\beta_{1}$ is the first characteristic number and $\left(t^{n}, \varphi\right)$ is equisingular, $\varphi(t)=Q(t)+\Sigma_{i \geqslant \beta_{1}} a_{i} t^{i}$, where $a=a_{\beta_{1}}$ is a unit and 
$Q(t)=\Sigma_{n j<\beta_{1}} a_{n j} t^{n j} \in B$. Let $\varphi_{1}=a^{-1}(\varphi-Q) \in B$. Consider the first exponent $r$ such that $r \notin R, a_{r} \neq 0$ and $r<d$. Then $r \in S(c)$; hence, there is $\alpha=$ $a_{r} t^{r}+\ldots \in B$. Then in $\varphi_{1}-\alpha$ the coefficients of $t^{j}(j<r)$ are the same in $\varphi_{1}$, and the coefficient of $t^{r}$ is zero. Continuing in this way, after finitely many steps, we get $\beta \in B$, such that $\varphi_{1}-\beta=P+\gamma$, where $o(\gamma) \geqslant c$. But, by 2.12, $\gamma \in B$ and, hence, $P \in B$. Thus $P=\Sigma a_{i j}\left(t^{n}\right)^{i} \varphi^{j}$, and a simple argument with orders shows that $a_{01}$ is a unit and, hence, there is a series $\Lambda(x, y)=\Sigma b_{i j} x^{i} y^{j}$, $b_{i j} \in A$, such that $\varphi=\Lambda\left(t^{n}, P\right)$. Hence, $A\left[\left[t^{n}, P\right]\right]=B$. The proof of the uniqueness of $P$ is also elementary and we omit it. To see (b) it suffices to take the automorphism defined by $(x, \Lambda(x, y))$.

Given a characteristic $\mathrm{c}=\left(n ; \beta_{1}, \ldots, \beta_{g}\right)$, we shall write $R(\mathrm{c})=\{i \in$ $\left.Z / \beta_{1}<i<d, i \notin S(\mathrm{c})\right\}$, where $d=$ degree of the conductor of $\mathrm{c}$.

3. A theorem on truncations. In this section we shall use a more geometric language. The category of affine schemes of the form $\operatorname{Spec}(A)$, where $A \in \mathrm{A}_{c}$, with the usual morphisms of schemes will be denoted by $S$.

Definition 3.1. A formal family of plane branches is a system $f=(\pi, X$, $Y, s)$ (where $Y=\operatorname{Spec}(A) \in S, \pi: X \rightarrow Y$ and $s: Y \rightarrow X$ are morphisms) such that for some $f \in A[[x, y]]$ (with $f(0,0)=0$ and $\operatorname{res}(f)$ irreducible) there is a commutative diagram

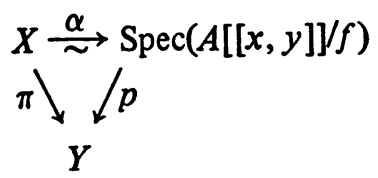

( $\alpha$ an isomorphism); moreover, if $s^{\prime}$ is the trivial section of $p$, then $\alpha s=s^{\prime}$. We call $X$ and $Y$ the total and parameter space of $\mathcal{F}$, respectively.

For the rest of this section, the term "family" shall mean "formal family of plane branches".

DEFinition 3.2. If $f$ in diagram (1) is an equisingular deformation of $f_{0}=$ $\operatorname{res}(f)$, then the family is called equisingular; the characteristic of $f$ is called the characteristic of the family. If $\mathfrak{z}$ is an equisingular family of characteristic c, we shall say that $f$ is a c-family.

DEFinition 3.3. We keep the notation of 3.1. The $\nu$ th truncation of a family $\mathcal{F}$ is the system $\mathfrak{F}_{\nu}=\left(\Pi_{\nu}, X_{\nu}, Y, s_{\nu}\right)$ where $X_{\nu}$ is the subscheme of $X$ defined by $I^{\nu+1}\left(I=\right.$ ideal defining $s(Y)$ ) and $\Pi_{\nu}: X_{\nu} \rightarrow Y$ and $s_{\nu}: Y \rightarrow X_{\nu}$ are induced by $\pi$ and $s$, respectively.

An isomorphism of families $\mathfrak{F}_{i}=\left(\pi_{i}, X_{i}, Y, s_{i}\right), i=1,2$, is an $Y$-isomorphism $X_{1} \rightarrow X_{2}$ commuting with $\pi_{i}$ and $s_{i}, i=1,2$. Isomorphisms of $\nu$-truncations are defined similarly.

THEOREM 3.3. Given a characteristic $\mathrm{c}=\left(n ; \beta_{1}, \ldots, \beta_{g}\right)$, there is an inte- 
ger $\nu_{0} \geqslant 0$ with the following property: if $\mathfrak{F}, G$ are c-families with the same parameter space $Y$, and if for some $\nu \geqslant \nu_{0}$ there is an isomorphism of v-truncations, then $\mathcal{F}$ is isomorphic to $G$.

REMARK 3.4. Note that the number $\nu_{0}$ depends on the characteristic c only. This theorem is well known when the parameter space of $\mathcal{F}$ and $G$ is a point [7, Theorem A]. Our proof follows closely the classical one, using the results of $\S \S 1$ and 2.

Before the proof of 3.3, we need some preliminary results.

RemarK 3.5. Recall that given $Z=\operatorname{Spec}(A[[x, y]]), A \in A_{c}$, we can take the blow up of $Z$ with center $Y$, the subscheme defined by the ideal $(x, y)$. It is the union of two affines $Z_{i}=\operatorname{Spec}\left(A\left[\left[x_{i}, y_{i}\right]\right]\right), i=1,2$, conveniently patched. Given $f \in A[[x, y]], X$ the subscheme of $Z$ defined by $(f)$, we can take the proper transform of $X$, which is defined over $Z_{1}$ by $x_{1}^{-s} f\left(x_{1} y_{1}, y_{1}\right)$, over $Z_{2}$ by $y_{2}^{-s} f\left(x_{2}, x_{2} y_{2}\right)$, where $s=o(f)$. Assume now that $f$ is a deformation of an irreducible $f_{0} \in k[[x, y]]$, admitting a parametrization $\left(t^{n}, \varphi\right), \varphi=a_{m} t^{m}+\ldots$, $m \geqslant n$. Note that then $n=o(f)=o(f(0, y))$. Consider $f_{1}\left(x_{1}, y_{1}\right)=$ $x_{1}^{-n} f\left(x_{1} y_{1}, y_{1}\right)$. If $m>n$, this power series has no constant term. If $m=n$, we have $f_{1}\left(0, a_{n}\right)=0$. In fact, from $f\left(t^{n}, \varphi\right)=0$ we get $t^{n} f_{1}\left(t^{n}, t^{-n} \varphi(t)\right)=0$; hence, $f_{1}\left(t^{n}, t^{-n} \varphi(t)\right)=0$ and, making $t=0$, we have $f_{1}\left(0, a_{n}\right)=0$. So in any case we can write $f_{1}\left(x_{1}, y_{1}\right)=f^{\prime}\left(x_{1}, y_{1}-a_{n}\right)$. We note: (a) $f^{\prime}\left(x^{\prime}, y^{\prime}\right)$ is a deformation of the proper transform of $f_{0}$, (b) $f_{1}$ admits the parametrization $\left(t^{n}, \varphi^{\prime}\right)$, where $\varphi^{\prime}(t)=t^{-n} \varphi(t)-a_{n}$. The series $f_{1}$, together with this parametrization, is called the parametrized transform of the curve $f$ (with the parametrization $\left(t^{n}, \varphi\right)$ ).

Similar definitions are given if $f$ admits a parametrization $\left(\psi, t^{n}\right), o(\psi) \geqslant n$.

Next we prove two elementary lemmas which are used in the proof of Theorem 3.3.

LEMMA 3.6. Let $f \in A[[x, y]]$ be a deformation of an irreducible $f_{0} \in$ $k[[x, y]]$, assume $f$ admits a strict equisingular parametrization $\left(t^{n}, \varphi\right)$ (see 1.3(ii)), and let $f^{\prime}$, parametrized by $\left(t^{n}, \varphi^{\prime}\right)$ be the parametrized transform of $f$. If $o\left(\varphi^{\prime}\right)<n$, then the initial coefficient of $\varphi^{\prime}$ is a unit.

Proof. Let the characteristic of $\left(t^{n}, \varphi\right)$ be $\left(n ; \beta_{1}, \ldots, \beta_{g}\right)$ and $\varphi=$ $\sum_{i=m}^{\infty} a_{i} t^{i}$ ( $a_{m}$ a unit). If $m>n$, then $\varphi^{\prime}=\sum_{i=m}^{\infty} a_{i} t^{i-n}$, hence, the leading coefficient is again $a_{m}$, a unit. If $n=m$, then we claim that in $\varphi, a_{i}=0$ for $n<$ $i<\beta_{1}$. In fact, assume by contradiction that $l$ is the first such index with $a_{l} \neq$ 0 . Since $\beta_{1}$ is the second characteristic number, $l=q n, q \geqslant 2$. Then, as we have $\varphi^{\prime}(t)=t^{-n} \varphi(t)-a_{n}=a_{l} t^{(q-1) n}+\ldots$, by our assumption $o\left(\varphi^{\prime}\right)<n$ we get $(q-1) n<n$; hence, $q<2$, a contradiction. Hence $\varphi^{\prime}(t)=a_{\beta_{1}} t^{\beta_{1}-n}+\ldots$, and $a_{\beta_{1}}$ is a unit (by equisingularity). 
LEMMA 3.7. Let $f=\Sigma a_{i j} x^{i} y^{i}$ be a deformation (over $A \in A_{c}$ ) of an irreducible $f_{0} \in k[[x, y]]$, let $\left(t^{n}, \varphi\right), \varphi=a_{m} t^{m}+\ldots, m \geqslant n$, be a parametrization of $f$. Then: (1) $a_{0 n}$ is a unit of $A$; (2) $a_{n}=n^{-1} a_{0 n} a_{1, n-1}$.

Proof. Write $f=u h$, where $k$ is the series associated to $f$, and $u$ a unit in $A[[x, y]]$ (use Remark 1.10). Then (1) and (2) easily follow by comparing the forms of lowest degree of $f$ and $u h$.

REMARK 3.8. Recall that, given a branch $f_{0}$, the set $\{n \in Z /$ a sequence of quadratic transforms of length $n$ desingularizes $f_{0}$ \} is nonempty. Its minimum is denoted $\sigma\left(f_{0}\right)$. Actually, this number depends on the equivalence class of $f_{0}$, i.e., given branches $f_{0}, g_{0}$ such that $\operatorname{ch}\left(f_{0}\right)=\operatorname{ch}\left(g_{0}\right)$, then $\sigma\left(f_{0}\right)=\sigma\left(g_{0}\right)$. Thus, we may define $\sigma(\mathbf{c})$, for a characteristic $\mathrm{c}$, as $\sigma\left(f_{0}\right)$, where $f_{0}$ is any branch such that $\operatorname{ch}\left(f_{0}\right)=$ c. See [15] for more details.

LEMMA 3.9. Given a characteristic $\mathrm{c}=\left(n ; \beta_{1}, \ldots, \beta_{g}\right)$ and an integer $s$, there is a number $r$ (depending on $\mathrm{c}$ and $s$ ) such that if $f, g$ are equisingular deformations of c-branches $f_{0}, g_{0}$, respectively, admitting strict parametrizations $\left(t^{n}\right.$, $\psi),\left(t^{n}, \omega\right)$ (with $\left.o(\psi) \geqslant n, o(\omega) \geqslant n\right)$, then a congruence $f \equiv g \bmod (x, y)^{r}$ implies the existence of parametrizations $\psi^{\prime}, \omega^{\prime}$ associated with $\psi, \omega$, respectively, such that $\psi^{\prime} \equiv \omega^{\prime}\left(\bmod t^{5}\right)$.

Proof. The proof is by induction on $\sigma(c)$. If $\sigma(c)=0$, then the curves $f_{0}$, $g_{0}$ are nonsingular and the lemma is easy, by solving $y$ in terms of $t=x$.

Assume 3.9 true for characteristic with $\sigma<p$. Consider $\mathrm{c}$ with $\sigma(\mathrm{c})=$ $p>0$. If $f \equiv g \bmod (x, y)^{n+1}$, then necessarily the $n$th coefficients of $\psi$ and $\omega$ are equal, say to $a \in A$. In fact, if $h_{q}$ denotes the sum of homogeneous terms of degree $q$ of a series $h$, we have $f_{n}=g_{n}$ and $a=-n^{-1}$ (coefficient of $x y^{n-1}$ in $f_{n}$ ) (cf. Lemma 3.7). Thus the proper transforms of $f$ and $g$ will be $f_{1}\left(x_{1}, y_{1}\right)$ and $g_{1}\left(x_{1}, y_{1}\right)$, respectively, which admit parametrizations $\left(t^{n}, \psi_{1}\right),\left(t^{n}, \omega_{1}\right)$, where $\psi_{1}(t)=\psi(t) / t^{n}-a, \omega_{1}(t)=\omega(t) / t^{n}-a$. By Lemma 3.6 the initial coefficients of $\psi_{1}$ and $\omega_{1}$ are units. These are equisingular parametrizations (clear if $o(\psi) \geqslant 2 n$, otherwise a consequence of the inversion formula), and if $c^{\prime}=$ $\operatorname{ch}\left(f_{1}\right)$, then $\sigma\left(\mathbf{c}^{\prime}\right)<\sigma(\mathbf{c})$.

Given the integer $s$, let $r^{\prime}$ be the number that, by induction, corresponds to $\mathbf{c}^{\prime}$. We claim that $r=r^{\prime}+n$ satisfies the requirements for $\mathbf{c}$ (and note that $\mathbf{c}^{\prime}$ depends only on c, not on the particular curve $f)$. In fact, if $f \equiv g \bmod (x, y)^{r^{\prime}+n}$, then $f_{1} \equiv g_{1} \bmod \left(x_{1}, y_{1}\right)^{r^{\prime}}$. Then by induction there are parametrizations $\left(t^{n}\right.$, $\left.\psi_{1}^{\prime}\right),\left(t^{n}, \omega_{1}^{\prime}\right)$ associated with $\psi_{1}$ and $\omega_{1}$, respectively, satisfying $\omega_{1}^{\prime} \equiv$ $\psi_{1}^{\prime} \bmod t^{s-n}$ (clear if $o\left(\psi_{1}\right) \geqslant n$, otherwise by induction and the argument in the proof of 1.5). Then $\left(t^{n}, t^{n}\left(\psi_{1}^{\prime}+a\right)\right),\left(t^{n}, t^{n}\left(\omega_{1}^{\prime}+a\right)\right)$ are parametrizations of $f, g$, respectively, congruent $\bmod \left(t^{s}\right)$, and associated with $\psi$ and $\omega$, respectively 
(by Proposition 1.11). This proves the lemma.

Proof of Theorem 3.3. Let the total spaces of $\mathfrak{F}$ and $G$ correspond to rings $B=A[[x, y]] /(f)$ and $B^{\prime}=A[[x, y]] /(g)$, respectively (cf. Definition 3.1, especially diagram (1) there). Consider an integer $\nu \geqslant 0$ and assume there is an isomorphism of truncations $\mathfrak{F}_{\nu} \approx G_{\nu}$. It is easy to verify that the isomorphism $\mathfrak{F}_{\nu} \approx G_{\nu}$ implies the existence of an automorphism $\phi$ of $A[[x, y]]$ and a unit $u \in$ $A[[x, y]]$ such that $\phi(f) \equiv u \cdot g \bmod (x, y)^{\nu+1}$.

Thus we may assume (changing the notation if necessary) that $f \equiv$ $g \bmod (x, y)^{\nu+1}$. Write $f_{0}$ (resp. $g_{0}$ ) for $f($ resp. $g$ ) reduced $\bmod M$, where $M=$ $r(A)$. Then we also may assume (after a linear change of coordinates, if necessary) that the $y$-axis is neither tangent to $f_{0}$ nor $g_{0}$, and $f, g$ are equisingular deformations of $f_{0}, g_{0}$, respectively. Now take $\nu \geqslant n$. With this choice, we may also assume that $f, g$ admit equisingular parametrizations $\left(t^{n}, \psi\right),\left(t^{n}, \omega\right)$ and both of them are strict. (To see this, for instance, we can assume that the $x$-axis is neither tangent to $f_{0}$ nor $g_{0}$. Hence, this series has terms $x^{n}, y^{n}$ with nonzero coefficients, and any parametrizations $\left(t^{n}, \psi\right),\left(t^{n}, \omega\right)$ of $f, g$, respectively, must satisfy $n=o(\psi)=\bar{o}(\psi)=o(\omega)=\bar{o}(\omega)$.)

Now apply Lemma 3.9 with $s=d$; write $\nu_{0}=r$. Consider $\nu \geqslant \nu_{0}$; then, by Lemma 3.9 , the series $f$ (resp. $g$ ) has a parametrization $\left(t^{n}, \psi_{1}\right)$ (resp. $\left(t^{n}, \omega_{1}\right)$ ) with $\omega_{1} \equiv \psi_{1}\left(\bmod t^{d}\right)$. By Theorem $1.9, B \approx A\left[\left[t^{n}, \psi_{1}\right]\right], B^{\prime} \approx A^{\prime}\left[\left[t^{n}, \omega_{1}\right]\right]$. We claim the right-hand sides are equal. In fact, write

$$
\psi_{1}(t)=P(t)+\sum_{i=d}^{\infty} a_{i} t^{i}, \quad \omega_{1}(t)=P(t)+\sum_{i=d}^{\infty} b_{i} t^{i}
$$

with $P$ a polynomial of degree less than $d$. But by Corollary 2.12 the series $N=$ $\Sigma_{i=d}^{\infty} a_{i} t^{i}$ and $N^{\prime}=\Sigma_{i=d}^{\infty} b_{i} t^{i}$ are in $B$ and $B^{\prime}$, respectively. Hence, $A\left[\left[t^{n}, \psi_{1}-N\right]\right]=A\left[\left[t^{n}, \omega_{1}-N^{\prime}\right]\right]=A\left[\left[t^{n}, P\right]\right]$, and $B$ is $A$-isomorphic to $B^{\prime}$. It follows that $\mathcal{F}$ is isomorphic to $G$.

Definition 3.10. Given a characteristic c, its $e$-number (denoted $e(\mathrm{c})$ ) is the least integer $\nu_{0}$ satisfying the condition of Theorem 3.3.

REMARK 3.11. Clearly, if we deal with nonsingular curves $(\sigma(c)=0)$ then $e=0$. The converse is not true. For instance, if $c=(2 ; 3), e(c)=0$. (It is a characteristic with "no moduli" [3, §4].)

Definition 3.12. (a) We say that a characteristic c "has no moduli" if, given an algebraically closed field $k$, and any two branches of characteristic $\mathrm{c}$, then they are isomorphic over some extension of $k$. By Krull's generalization of the Nullstellensatz [7, p. 365], if $k$ is algebraically closed, uncountable, then the isomorphism can be taken over $k$. An example is $\mathrm{c}=(2,2 n+1), n$ any nonnegative integer, or $(4 ; 6,2 n+1), n \geqslant 3[3, \S 4]$.

(b) A family $\mathfrak{F}$ is trivial if it is isomorphic to a family $\operatorname{Spec}(B) \rightarrow \operatorname{Spec}(A)$, 
where $B=A[[x, y]] /\left(f_{0}\right), f_{0} \in k[[x, y]]$.

Proposition 3.13. Let $\mathrm{c}$ be a characteristic. Then the following are equivalent: (1) c has no moduli.

(2) Any c-family is trivial.

(3) $e(\mathrm{c})=0$.

Proof. The equivalence of (2) and (3) is trivial. To show that (1) implies (2), first assume the family $\mathfrak{F}$ has a nonsingular parameter space. By equisingularity, the special and generic curves of $\mathcal{F}$ are equivalent (cf. 2.7(a)), hence, by (1), isomorphic. But Seidenberg proved [11] that in this situation the family is trivial. The general case reduces to this one by using Lemma 2.5. Finally, to check that (2) implies (1), take two branches $X_{i}, i=1,2$, of the same characteristic $\mathrm{c}=\left(n ; \beta_{1}, \ldots, \beta_{g}\right)$. By Corollary 2.13 (with $A=k$ ) we may assume they correspond to parametrizations:

$$
\left(x=t^{n}, y=t^{n}+\sum_{j \in R} \lambda_{j}^{i} t^{j}\right), \quad i=1,2, \quad R=R(\mathrm{c}) .
$$

Let $\left\{z_{j}\right\}, j \in R$, be analytic indeterminates; then the families

$$
\operatorname{Spec} A_{i}\left[\left[t^{n}, t^{\beta_{1}}+\sum_{j \in R} z_{j} t^{j}\right]\right] \rightarrow \operatorname{Spec}\left(A_{i}\right),
$$

where $A_{i}=k\left[\left[\left\{z_{j}-\lambda_{j}^{i}\right\}\right]\right], j \in R, i=1,2$, are equisingular and the special curves are isomorphic to $X_{i}, i=1,2$, respectively. By (2), these families are trivial, hence $X_{i}(i=1,2)$ is isomorphic (over an extension of $\left.k\right)$ to the curve parametrized by $\left(t^{n}, t^{\beta}+\Sigma_{j \in R} z_{j} t^{t}\right)$.

4. The complex analytic case. In this section we see that the formal results of the previous sections remain valid in the analytic (convergent) case. Now we work over the field $C$ of complex numbers. The ring of complex convergent power series will be denoted by $C\left\{x_{1}, \ldots, x_{n}\right\}$. By an analytic ring we mean a ring of the form $C\left\{x_{1}, \ldots, x_{n}\right\} / I, I$ an ideal. Thus, an analytic ring is a local $C$-algebra in a natural way. The category whose objects are analytic rings, and morphisms are $C$-algebra homomorphisms, sending identities to identities (they are necessarily local) is denoted by $H$. In this and the next section, a branch shall mean a germ of a plane analytic curve, irreducible at its center (or, by abuse of language, its local ring). If $f_{0} \in C\{x, y\}$ and is irreducible, the expression "the branch $f_{0}$ " means the germ, at the origin, of the curve locally defined by $f_{0}$. Branches in the sense of $\S 0$ will be called, from now on, formal branches.

Remark 4.1. Let $A \in H, A=C\left\{u_{1}, \ldots, u_{r}\right\} / I, \Pi: C\{u\} \rightarrow A$ be the canonical map. Consider the following subset $B$ of $A\left[\left[x_{1}, \ldots, x_{n}\right]\right]$. A series $f \in$ 
$B$ if it is the image of some $g \in C\left\{u_{1}, \ldots, u_{r}, x_{1}, \ldots, x_{n}\right\}=R$ under the canonical map $R \rightarrow A\left[\left[x_{1}, \ldots, x_{n}\right]\right]$ (i.e., $\left.\Sigma a_{\alpha}(u) x^{\alpha} \rightarrow \Sigma \Pi\left(a_{\alpha}(u)\right) x^{\alpha}\right)$. It is easy to see that $B$ is independent of the representation $A=C\left\{u_{1}, \ldots, u_{n}\right\} / I$ that we chose. It is a subring of $A\left[\left[x_{1}, \ldots, x_{n}\right]\right]$ and is an analytic ring. We shall denote it by $A\left\{x_{1}, \ldots, x_{n}\right\}$. Note that if $(X, x)$ is an analytic germ, with $\mathrm{O}_{X, x} \approx A$, then $A\left\{x_{1}, \ldots, x_{n}\right\} \approx 0_{X \times A^{n},(x, 0)}\left(0=\right.$ origin of $\left.A^{n}\right)$.

REMARK 4.2. We shall need the following facts on analytic rings:

(a) If $A \in H$, and $u \in A$ is a unit, then $u$ has $n$th roots in $A$ for any positive integer $n$.

(b) Analytic rings are noetherian, excellent, henselian local rings.

(c) Let $A \in H$ be reduced (i.e., without nonzero nilpotents); then $A$ is integrally closed in its completion.

(d) Let $A \in H, f \in A\{x, y\}, \varphi, \psi$ be in $A\{t\}$. Then the series $f(\varphi, \psi) \in$ $A[[t]]$ is in $A\{t\}$.

(e) Given $\varphi, \psi$ in $A\{t\}$, there is a unique homomorphism $p: A\{x, y\} \rightarrow$ $A\{t\}$ satisfying $p(x)=\varphi, p(y)=\psi$. The image of $p$ will be denoted $A\{\varphi, \psi\}$. This is an analytic ring.

Points (a) and (b) are well known (see, e.g., [5]). Point (c) is a consequence of $[9$, Theorem 3,126$]$, which says that an excellent henselian reduced ring is integrally closed in its completion. Points (d) and (e) are easily verified.

With Remarks 4.1 and 4.2(a), (d), it is clear how to obtain the complex analogues of (1.1) to (1.5). To extend other results we need

LEMMA 4.3. Let $A$ be an analytic ring, $\varphi, \psi$ (elements of $A\{t\}$ ) a parametrization of $f \in A\{x, y\}$. Then:

(a) $A\{t\}$ is a finite $A\{\varphi, \psi\}$-algebra.

(b) $(A\{\varphi, \psi\})^{\wedge} \approx \hat{A}[[\varphi, \psi]]$ ( indicates completion with respect to the maximal ideal).

Proof. We may assume (by analytic (1.5)) that $\varphi=t^{n}$. Then (a) is clear, since $A\{t\}$ is finite over $A\left\{t^{n}\right\}$.

To see (b), consider the exact sequence $0 \rightarrow P \rightarrow A\{x, y\} \stackrel{\alpha}{\rightarrow} A\{t\}$ (where $\alpha(x)=\varphi, \alpha(y)=\psi)$. Tensoring with $\hat{A}[[x, y]]$, and using the fact that $A\{t\}$ is $A\{\varphi, \psi\}$-finite, we have an exact sequence

$$
0 \rightarrow \mathrm{P} \hat{A}[[x, y]] \rightarrow \hat{A}[[x, y]] \stackrel{\hat{\alpha}}{\longrightarrow} \hat{A}[[t]],
$$

$\hat{\alpha}(x)=\varphi=t^{n}, \hat{\alpha}(y)=\psi$. From this the assertion easily follows.

THEOREM 4.4. Let $f$ be a deformation of an irreducible $f_{0} \in C\{x, y\}$ to $A \in H$ which admits a parametrization $x=\varphi(t), y=\psi(t)$, let $p: A\{x, y\} \rightarrow A\{t\}$ send $x$ into $\varphi, y$ into $\psi$. Then (a) $(f)=\operatorname{ker}(p),(\mathrm{b}) A\{\varphi, \psi\}$ is $A$-flat.

Proof. Consider the sequence of $A\{x, y\}$-modules 


$$
0 \rightarrow(f) \stackrel{j}{\rightarrow} A\{x, y\} \stackrel{p}{\rightarrow} A\{t\},
$$

where $j$ is the inclusion. By 4.3(a), and the fact that analytic rings are noetherian, all these are finite $A\{x, y\}$-modules. Hence, tensoring with $\hat{A}[[x, y]]$ gives

$$
0 \rightarrow(f) \rightarrow \hat{A}[[x, y]] \stackrel{\hat{p}}{\rightarrow} \hat{A}[[t]] .
$$

By 1.9 , this sequence is exact. Hence, since $\hat{A}[[x, y]]$ is faithfully flat over $A\{x, y\},(1)$ is already exact, i.e., (a) is proved.

(b) is a general consequence of the fact that if $B$ is local noetherian, then $B \rightarrow \hat{B}$ is faithfully flat.

With this it is easy to obtain the complex analogue of the remaining results of $\S 1$ and all the results of $\S 2$ from 2.1-2.5. The definition of the semigroup $S(A\{x, y\} /(f))$ is similar to the formal case and the analogue of Theorem 2.11 holds. In fact, its proof, with minor changes, can be applied to the complex case too. (Use the obvious fact that $S(A\{x, y\} /(f)) \subset S\left(\hat{A}[[x, y]] /\left(f_{0}\right)\right)$, with equality if $A=C$. Note that after we proved the analytic version of 2.11 , we can easily see that this inclusion is an equality for any $A \in H$.) Corollary 2.12 also holds, but it is not clear that the same proof can be used. We prove it in a different way.

Proposition 4.5. Let $f \in A\{x, y\}$ be an equisingular deformation of the branch $f_{0}$ which admits a parametrization $\left(t^{n}, \varphi\right)$. Let $\varphi_{0}$ be the image of $\varphi$ in $C\{t\}, d$ the degree of the conductor of $C\{t\}$ in $C\left\{t^{n}, \varphi_{0}\right\}$. Then any series $g \in$ $A\{t\}$ of order $\geqslant d$ is in $A\left\{t^{n}, \varphi\right\}$.

Proof. By writing $A=C\left\{u_{1}, \ldots, u_{r}\right\} / I$ and lifting $\left(t^{n}, \varphi\right)$ to an equisingular parametrization over $C\left\{u_{1}, \ldots, u_{r}\right\}$, one is reduced to the case when $A$ is a regular ring. So assume $A=C\left\{u_{1}, \ldots, u_{r}\right\}$. Let $g \in A\{t\}$ of order $\geqslant d$. As we saw (in 4.3(a)), $g$ is integral over $A\left\{t^{n}, \varphi\right\}$. Also, by 2.12, as an element of $\hat{A}[[t]], g \in \hat{A}\left[\left[t^{n}, \varphi\right]\right]=\left(A\left\{t^{n}, \varphi\right\}\right)$ :

By using Remark 4.2(d), we conclude $g \in A\left\{t^{n}, \varphi\right\}$.

Corollary 2.13 extends without problem to the analytic case.

The results of $\S 3$ hold, with minor changes (e.g., in Definition 3.1), in the complex case. We omit the details.

5. Versal analytic equisingular deformations. In this section, we show that any analytic branch has a versal equisingular deformation, in the following sense:

Definition 5.1. Let $f_{0} \in C\{x, y\}$ be irreducible. A pair $(E, h)$, where $E \in$ $H$ and $h \in E\{x, y\}$ is an equisingular deformation of $f_{0}$, is called a versal equisingular deformation if (a) given homomorphisms (in H) $\alpha_{i}: A \rightarrow A^{\prime}=A / I, \varphi^{\prime}$ : $E \rightarrow A^{\prime}$ and an equisingular deformation $f$ of $f_{0}$ over $A$ such that $\alpha^{*}(f)$ is simi- 
lar to $\varphi^{\prime *}(h)$, then there is a homomorphism $\varphi: E \rightarrow A$ such that $\varphi^{*}(h)$ is similar to $f$ and $\alpha \varphi=\alpha^{\prime}$. (b) In the special case when $A=k[\epsilon]$ (dual numbers over $k$ ) and $I=(\epsilon)$ (i.e., $\left.A^{\prime}=k\right), \varphi$ is uniquely determined.

Of course, there is a similar formal definition working over $A_{c}$. In this formal case the existence of a versal equisingular deformation follows from the results of [10] and [13] (cf. Corollary 2.9). One could try to show the existence of an analytic equisingular deformation by proving the convergence of the formal one, but this probably leads to complicated computations. In this section we will show directly the existence of the analytic deformation which can be done elementarily using the results of $\S 4$. Moreover, the process (which works in the formal case too) could have some independent interest.

To do this we need some previous remarks and lemmas. In the sequel we use primes to indicate the analytic version of a result of $\S \S 1$ or 2, e.g., Theorem (1.9)' means the analytic version of Theorem 1.9.

REMARK 5.2. There is a definition similar to 5.1, but without the equisingular condition. In this case it is known that an analytic versal deformation $\left(D, h_{1}\right)$ exists; moreover, $D$ is a regular ring ([6], [12]). In both cases the definition of versality is weaker than that of 5.1 (namely in (5.1)(a) is assumed that $I=r(A)$ and $A^{\prime}=C$ ), and in [12] only regular "parameter" rings are used, but these results will be sufficient for our needs.

LEMMA 5.3. Let $\mathrm{c}=\left(n ; \beta_{1}, \ldots, \beta_{g}\right)$ be a characteristic, $R(\mathrm{c})=R=\left\{i_{1}\right.$, $\left.\ldots, i_{m}\right\}, f_{0} \in C\{x, y\}$ associated with the parametrization $\left(t^{n}, t^{\beta_{1}}+\Sigma_{i \in R} \lambda_{i} t^{i}\right)$ and assume $\operatorname{ch}\left(f_{0}\right)=\mathrm{c}$. Let $B=C\left\{z_{i_{1}}-\lambda_{i_{1}}, \ldots, x_{i_{m}}-\lambda_{i_{m}}\right\}, g_{1} \in B\{x, y\}$ be associated with $\left(t^{n}, t^{\beta_{1}}+\Sigma_{i \in R} z_{i} t^{t}\right)$. Then $g_{1}$ is an equisingular deformation of $f_{0}$ over $B$, and $\left(B, g_{1}\right)$ satisfies Definition 5.1(a).

Proof. It is obvious that $g_{1}$ is equisingular. To check (a), let $\psi^{\prime}: B \rightarrow A^{\prime}$, $\alpha: A \rightarrow A^{\prime}$ and $f \in A\{x, y\}$ (an equisingular deformation of $f_{0}$ ) be given and assume $\psi^{\prime *}(g)$ and $\alpha^{*}(f)$ are similar. We may assume, after replacing $f$ by its similar $u f$ ( $u$ a suitable unit in $A\{x, y\}$ ), that (writing $f^{\prime}=\alpha^{*}(f)$ ) there is an automorphism of $A^{\prime}\{x, y\}$ (given by $\varphi^{\prime}, \Lambda^{\prime} \in A^{\prime}\{x, y\}$ ) such that $f^{\prime}\left(\varphi^{\prime}(x, y), \Lambda^{\prime}(x, y)\right.$ ) has the parametrization $\left(t^{n}, P^{\prime}(t)=t^{\beta_{1}}+\Sigma_{i \in R} a_{i}^{\prime} t^{i}\right)$, where $a_{i}^{\prime}=\psi^{\prime}\left(z_{i}\right)$. Lift $\left(\varphi^{\prime}, \Lambda^{\prime}\right)$ to an automorphism $\varphi, \Lambda$ of $A\{x, y\}$ and consider $f_{1}=f(\varphi, \Lambda)$. Let $\left(t^{n}\right.$, $\varphi$ ) be a parametrization of $f_{1}$. We may assume (after replacing, if necessary, $t$ by $\delta t, \delta^{n}=1$, by using Proposition $\left.(1.11)^{\prime}\right)$ that $\varphi$ reduces to $P^{\prime}$ over $A^{\prime}$. Thus, we get a homomorphism $A\left\{t^{n}, \varphi\right\} \stackrel{q}{\rightarrow} A^{\prime}\left\{t^{n}, P^{\prime}\right\}$. Using (2.13)', we write $A\left\{t^{n}, \varphi\right\}=$ $A\left\{t^{n}, P\right\}$, with $P(t)=t^{\beta_{1}}+\Sigma_{i \in R} a_{i} t^{i}$. By the uniqueness assertion in (2.13)'(a), we get $q(P)=P^{\prime}$, i.e., $\alpha\left(a_{i}\right)=a_{i}^{\prime}, i \in R$. By $(2.13)^{\prime}(\mathrm{b}), f_{1}$ is similar to the series associated with $\left(t^{n}, P\right)$. Now it is clear that the homomorphism $\psi: B \rightarrow A$ defined by $\psi\left(z_{i}\right)=a_{i}$ satisfies the required conditions. 
Lemma 5.4. Let $f_{0}$ be an irreducible series in $C\{x, y\}, A$ a reduced ring in $H, f \in A\{x, y\}$ a deformation of $f_{0}$ over $A$. Assume that as a formal deformation $f$ is equisingular. Then as an analytic deformation $f$ is equisingular.

Proof. We may assume, interchanging $x$ and $y$ if necessary, that the $y$-axis is not tangent to $f_{0}$. By the preparation theorem we may also assume

$$
f(x, y)=y^{n}+A_{1}(x) y^{n-1}+\ldots+A_{n}(x) ; \quad A_{i}(x) \in A\{x\} .
$$

By assumption, $f$ has an equisingular parametrization $x=t^{n}, y=\varphi(t) \in$ $\hat{A}[[t]]$. It follows from (1) that $\varphi$ is integral over $A[t]$. Then, by Remark 4.2(c), $\varphi \in A\{t\}$. The lemma is now clear.

We need the following lemma on formal deformations.

LEMma 5.5. Let $f \in A[[x, y]]$ be a deformation of a formal branch $f_{0}$. Assume (a) $A$ is an integral domain, (b) the series $f$ (regarded as an element of $K[[x, y]]$, where $K$ is an algebraic closure of $A)$ is irreducible and $\operatorname{ch}\left(f_{0}\right)=\operatorname{ch}(f)$. Then $f$ is an equisingular deformation of $f_{0}$.

Proof. The proof is by induction on $\sigma\left(f_{0}\right)$ (cf. Remark 3.8). Assume $\sigma\left(f_{0}\right)=0$, i.e., $f_{0}$ is nonsingular. Then in $f(x, y)=\Sigma a_{i j} x^{i} y^{j}$ either $a_{10}$ or $a_{01}$ is a unit. If, say, $a_{01}$ is a unit, then by solving $y$ in terms of $x$ we get a parametrization which clearly is equisingular.

Assume now $\sigma\left(f_{0}\right)=r$ and the lemma true for $\sigma<r$. Let $\operatorname{ch}\left(f_{0}\right)=(n$; $\beta_{1}, \ldots, \beta_{g}$ ). We may assume (after a change of variables with coefficients in $A$ if necessary) that

$$
f=y^{n}+h, \quad o(h)>n .
$$

(In fact, clearly we may assume $f=f_{n}+\ldots$, where $f_{n}=a_{n 0} x^{n}+\ldots+y^{n}$, $a_{i j} \in A$. As $f \in K[[x, y]]$ is a branch, $f_{n}=(y-\alpha x)^{n}, \alpha \in K$. But then $n \alpha=$ $\pm a_{1, n-1}$, i.e., $\alpha \in A$. Now the contention is clear.) Consider the proper transform $f^{\prime}$ of $f$. It is a deformation of $f_{0}^{\prime}$ (the proper transform of $f_{0}$ ) "centered at the origin" (by (2)). Let $\left(t^{n}, \varphi\right),\left(t^{n}, \varphi_{0}\right)$ be parametrizations of $f$ and $f_{0}$, respectively. We distinguish two cases:

(a) $2 n \leqslant \beta_{1}$. In this case, $\left(t^{n}, t^{-n} \varphi\right.$ ) (resp. $t^{n}, t^{-n} \varphi_{0}$ ) is a parametrization of $f^{\prime}$ (resp. $\left.f_{0}^{\prime}\right)$, satisfying $O\left(t^{-n} \varphi\right) \leqslant n$ (resp. $\left.O\left(t^{-n} \varphi_{0}\right)<n\right)$, i.e. $\operatorname{ch}\left(t^{n}, t^{-n} \varphi\right)=$ $\operatorname{ch}\left(f^{\prime}\right)\left(\right.$ resp. $\left.\operatorname{ch}\left(t^{n}, t^{-n} \varphi_{0}\right)=\operatorname{ch}\left(f_{0}^{\prime}\right)\right)$. Then it is clear that $\operatorname{ch}\left(f^{\prime}\right)=\operatorname{ch}\left(f_{0}^{\prime}\right)$, and by induction (note $\sigma\left(f_{0}^{\prime}\right)<r$ ) $f^{\prime}$ is equisingular. Then, by Proposition 1.11, $\left(t^{n}, t^{-n} \varphi\right)$ is an equisingular parametrization; hence, $\left(t^{n}, \varphi\right)$ is an equisingular parametrization of $f$.

(b) $2 n>\beta_{1}$. In this case $t^{-n} \varphi(t)=a t^{\beta_{1}}+\ldots, 0 \neq a \in K$, and $t^{-n} \varphi(t)=$ $\lambda t^{\beta_{1}}+\ldots, \lambda \neq 0,0 \neq \lambda \in k$. By "inverting" (i.e., passing to parametrizations $\left(\psi(\tau), \tau^{\beta_{1}}\right),\left(\psi_{0}(\tau), \tau^{\beta_{1}}\right)$ of $f^{\prime}$ and $f_{0}^{\prime}$ and using the inversion formula) we see that $\operatorname{ch}\left(f^{\prime}\right)=\operatorname{ch}\left(f_{0}^{\prime}\right)$. Hence, by $1.11, \psi(\tau) \in A[[\tau]]$ and $\left(\psi(\tau), \tau^{\beta_{1}}\right)$ is an equisingu- 
lar parametrization. Then clearly its "inversion" $\left(t^{n}, t^{-n} \varphi\right)$ is a parametrization in the sense of Definition 1.3 and $\operatorname{ch}\left(t^{n}, t^{-n} \varphi\right)=\operatorname{ch}\left(t^{n}, t^{-n} \operatorname{res}(\varphi)\right)$. From this it follows that $\left(t^{n}, \varphi\right)$ is an equisingular parametrization of $f$, and the lemma is proved.

Finally we need the following well-known result:

Lemma 5.6. Let $A \in H, M=r(A), B=C\left\{x_{1}, \ldots, x_{n}\right\}, \alpha: A \rightarrow B$ be an injective homomorphism, and assume the induced linear map $\alpha^{\prime}: M / M^{2} \rightarrow N / N^{2}$ is injective. Then (i) $\alpha$ has a retraction, (ii) $\hat{\alpha}: \hat{A} \rightarrow \hat{B}$ is injective, (iii) $A$ is regular.

Now we are ready to prove

THEOREM 5.7. Let $f_{0} \in C\{x, y\}$ be irreducible. Then there is an analytic versal equisingular deformation $(E, h)$ where $E$ is a regular ring.

Proof. First of all, we may replace $f_{0}$ by $f_{0}^{\prime}$ such that $C\{x, y\} / f_{0} \approx$ $C\{x, y\} / f_{0}^{\prime}$ and work with this new $f_{0}^{\prime}$. Thus, we may assume $f_{0}$ to be as follows. Let $\mathrm{c}=\left(n ; \beta_{1}, \ldots, \beta_{g}\right)$ be the characteristic of $f_{0}, \dot{d}=$ degree of conductor of c, $S$ the semigroup associated with $\mathrm{c}$, and $R=\left\{i \in Z / \beta_{1}<i<d, i \notin S\right\}$. Then $f_{0}$ is associated (see (1.10)) with a parametrization

$$
x=t^{n}, \quad y=\sum_{i \in R} \lambda_{i} t^{i} .
$$

Let $\left(D, h_{1}\right)$ be a (nonequisingular) analytic versal deformation of $f_{0}$. Consider the couple $\left(B, g_{1}\right)$ of Lemma 5.3. Then by versality there is a homomorphism $\varphi: D \rightarrow B$ such that $\varphi^{*}\left(h_{1}\right)$ is similar to $g_{1}$. Let $P=\operatorname{Ker} \varphi, E=D / P, \Pi: D \rightarrow$ $E$ be the quotient map, $h=\Pi^{*}\left(h_{1}\right)$. We shall see that $(E, h)$ is equisingular versal.

To see that it is equisingular, by Lemma 5.4 it is enough to check that the formal deformation $(\hat{E}, h)$ is equisingular. By Lemma 5.5 , it is enough to check that $h \in K[[x, y]]$ ( $K$ an algebraic closure of $\hat{E}$ ) is irreducible, and $\operatorname{ch}(h)$ and $\operatorname{ch}\left(f_{0}\right)$ are the same. But let $j: E=D / g \rightarrow B$ be the injective map induced by $\varphi$. Then $j^{*}(h)$ is similar to $g_{1}$ which is an equisingular deformation of $f_{0}$. If $K_{1}$ is an algebraic closure of $B$, then it follows: (i) $h \in K_{1}[[x, y]]$ is irreducible, and (ii) $\operatorname{ch}(h)$ (computed using $K_{1}$ ) agrees with $\operatorname{ch}\left(f_{0}\right)$. It is clear that (i) and (ii) are still true for $h \in K[[x, y]]$. This shows that $h$ is equisingular.

Next, we see that $j: E \rightarrow B$ has a retraction $p$. By Lemma 5.6 it is enough to check that the linear map of tangent spaces $\left(N / N^{2}\right)^{\prime} \rightarrow\left(M / M^{2}\right)^{\prime}(N=\operatorname{rad}(B)$, $M-\operatorname{rad}(A)$, a prime denotes dual vector space) is surjective. By the isomorphism (natural in $R \in H)\left(\operatorname{rad} R /(\operatorname{rad} R)^{2}\right)^{\prime} \approx \operatorname{Hom}(R, C[\epsilon])$, it is enough to verify: given a homomorphism $\gamma: E \rightarrow C[\epsilon]$, then there is a homomorphism $\beta: B \rightarrow C[\epsilon]$ such that $\beta j=\gamma$. But consider the deformation (over $C[\epsilon]$ ) $\gamma^{*}(h)$. By Lemma 5.3, there is $\beta: B \rightarrow C[\epsilon]$ such that $\beta^{*}\left(g_{1}\right)$ is similar to $\gamma^{*}(h)$. Then $(\beta \varphi)^{*}\left(h_{1}\right)$ is similar to $(\gamma \Pi)^{*}\left(h_{1}\right)$. By the versality of $\left(D, h_{1}\right)$ (property (b) in the definition), 
$\gamma \Pi=\beta \varphi$, i.e., $\gamma \Pi=\beta j \Pi$. Hence, $\gamma=\beta j$ because $\Pi$ is surjective.

Now we check property (a) in Definition 5.1. Consider the diagram (with solid arrows)

$$
\begin{gathered}
E \stackrel{p}{\stackrel{p}{\longrightarrow}} B \\
\varphi / \varphi^{\prime} \downarrow \\
A \underset{\alpha}{\longrightarrow} A / I=A^{\prime}
\end{gathered}
$$

( $\alpha$ is the quotient map) and deformations $f$ (over $A$ ) and $f^{\prime}$ (over $A^{\prime}$ ) of $f_{0}$; we assume $f^{\prime}$ is similar to both $\alpha^{*}(f)$ and $\left(\varphi^{\prime}\right)^{*}(h)$. We want to construct $\varphi$ such that the diagram commutes and $\varphi^{*}(h)$ is similar to $f$. Let $\psi^{\prime}=\varphi p$. Then by Lemma 5.3 , there is $\psi: \beta \rightarrow A$ such that $\psi^{*}\left(g_{1}\right)$ is similar to $f$ and $\alpha \psi=\psi^{\prime}$. It is now clear that $\varphi=\psi j$ is a homomorphism as we wanted.

To verify property (b) (in Definition 5.1$)$ for $(E, h)$ we must verify: given $\gamma, \gamma^{\prime}: E \rightarrow C[\epsilon]$, if $\gamma^{*}(h)$ is similar to $\gamma^{\prime *}(h)$ then $\gamma=\gamma^{\prime}$. But if $\gamma^{*}(h)$ is similar to $\gamma^{*}(h)$, then $(\gamma \Pi)^{*}\left(h_{1}\right)$ is similar to $\left(\gamma^{\prime} \Pi\right)^{*}\left(h_{1}\right)$. By versality of $\left(D, h_{1}\right), \gamma \Pi=$ $\gamma^{\prime} \Pi$; hence, $\gamma=\gamma^{\prime}$ since $\Pi$ is surjective.

That $E$ is regular follows from Lemma 5.6. In fact, we saw already that the conditions of 5.6 hold for $j: E \rightarrow B$.

REMARK 5.8. The results presented in this section remain valid in the formal case over a field $k$ algebraically closed of characteristic zero. Precisely:

(a) There is a formal versal (not equisingular) deformation of a formal branch $f_{0}$. Moreover, over $C$, the ' pair $\left(\hat{D}, h_{1}\right)$ is such a versal object. (In fact, let $g_{1}, \ldots, g_{s}$ be elements of $k[[x, y]]$, inducing a basis of the vector space $k[[x, y]] / I$, where $I=\left(f_{0}, \partial f_{0} / \partial x, \partial f_{0} / \partial y\right)$. Then the deformation over $k\left[\left[t_{1}, \ldots, t_{s}\right]\right]$ of equation $f_{0}+\Sigma_{i=1}^{s} t_{i} g_{i}$ is versal. In the analytic case the construction is similar.) See [6] and [12].

(b) The formal versions of Lemmas 5.3, 5.6 and Theorem 5.7 remain valid with similar proofs. This gives a proof of the existence of a hull for the functor $P$ (see Corollary 2.9) independent of Schlessinger's criterion.

Now we want to compare the analytic and formal deformations of $f_{0}$.

THEOREM 5.9. Let $f_{0},(E, h)$ be as in Theorem 5.7. Then $(\hat{E}, h)$ is a formal versal equisingular deformation of $f_{0} \in C[[x, y]]$.

Proof. As in the proof of Theorem 5.7, we may assume that $f_{0}$ is associated to the parametrization (3) of that proof. Then, as remarked in 5.8(b), and using the notation of Theorem 5.7 , if $\hat{\varphi}: \hat{D} \rightarrow \hat{B}$ is induced by $\varphi$ by completing, $Q=\operatorname{Ker}(\varphi)$, and $\Pi_{1}: \hat{D} \rightarrow \hat{D} / Q=E^{\prime}$, then $\left(E^{\prime}, \Pi_{1}^{*}\left(h_{1}\right)\right)$ is a versal formal equisingular deformation of $f_{0}$. To prove the theorem it will suffice to verify $\hat{P}=P \hat{D}=$ $Q$. But we have a diagram 
<smiles>OP[Te]1[Te][Te](O)(O)[Te]1</smiles>

with exact row and column. Completing, and using Lemma 5.6(ii), we get a diagram with the same exactness properties

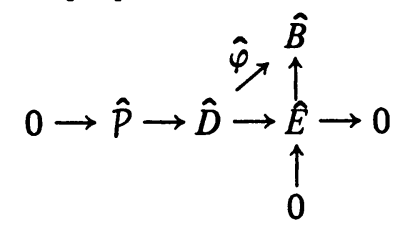

which shows that $\hat{P}=Q$. The theorem is proved.

\section{REFERENCES}

1. S. S. Abhyankar, On the ramification of algebraic functions, Amer. J. Math. 77 (1955), 575-592. MR 17, 193.

2. Inversion and invariance of characteristic pairs, Amer. J. Math. 89 (1967), 363-372. MR 36 \#3784.

3. S. Ebey, The classification of singular points of algebraic curves, Trans. Amer. Math. Soc. 118 (1965), 454-471. MR 31 \#1251.

4. H. Hironaka, On the equivalence of singularities. I, Arithmetical Algebraic Geometry (Proc. Conf., Purdue Univ., 1963), Harper \& Row, New York, 1965, pp. 153-200. MR 34 \#1317.

5. C. Hozuel, Séminaire Cartan 13ième année: 1960/61, Familles d'espaces complexes et fondements de la géométrie analytique, Exposés 18, 21, École Normale Supérieure, Secrétariat Mathématique, Paris, 1962. MR 26 \#3562.

6. A. Kas and M. Schlessinger, On the versal deformation of a complex space with an isolated singularity, Math. Ann. 196 (1972), 23-29. MR 45 \#3769.

7. W. Krull, Jacobsonche Ringe, Hilbertscher Nullstellensatz, Dimensionstheorie, Math. Z. 54 (1951), 354-387. MR 13, 903.

8. H. Matsumura, Commutative algebra, Benjamin, New York, 1970. MR 42 \#1813.

9. M. Raynaud, Anneaux locaux henséliens, Lecture Notes in Math., vol. 169, SpringerVerlag, Berlin and New York, 1970. MR 43 \#3252.

10. M. Schlessinger, Functors of Artin rings, Trans. Amer. Math. Soc. 130 (1968), 208-222. MR 36 \#184.

11. A. Seidenberg, Analytic products, Amer. J. Math. 91 (1969), 577-590. MR 40 \#261.

12. G. N. Tjurina, Locally semiuniversal flat deformations of isolated singularities of complex spaces, Izv. Akad. Nauk SSSR Ser. Mat. 33 (1969), 1026-1058 = Math. USSR Izv. 3 (1969), 967-1000. MR 40 \#5903.

13. J. Wahl, Equisingular deformations of plane algebroid curves, Trans. Amer. Math. Soc. 193 (1974), 143-170.

14. R. J. Walker, Algebraic curves, Princeton Math. Ser., vol. 13, Princeton Univ. Press, Princeton, N. J., 1950. MR 11, 387.

15. O. Zariski, Studies in equisingularity. I, II, III, Amer. J. Math. 87 (1965), 507536, 972-1006; ibid. 90 (1968), 961-1023. MR 31 \#2243; 33 \#125; 38 \#5775.

16. Contributions to the problem of equisingularity, Questions on Algebraic Geometry (C. I. M. E., II Ciclo, Varenna, 1969), Edizioni Cremonese, Rome, 1970, pp. 261343. MR 43 \#1 987. 